\title{
Periods of $\beta$ Cephei and SPB Stars from Hipparcos Photometry $^{1}$
}

\author{
J. Molenda-Żakowicz \\ Wroclaw University Observatory, ul. Kopernika 11, 51-622 Wroclaw, \\ Poland
}

\begin{abstract}
For the $\beta$ Cephei variables, we compare the periods derived from ground-based observations with those given in the Hipparcos catalogue. For the SPB stars, of which most have been discovered by Hipparcos, we examine the question of multiperiodicity.
\end{abstract}

\section{Periods of $\beta$ Cep Stars}

The Hipparcos satellite observed 43 out of $63 \beta$ Cephei stars listed in Sterken \& Jerzykiewicz (1993). Four of the 43 stars were classified in the Hipparcos catalogue as unsolved variables and six as microvariables. Hipparcos confirmed also that $\alpha \mathrm{Vir}$ is an ellipsoidal variable. In three cases, viz., $\mathrm{KK} \mathrm{Vel,} \kappa$ Sco and EN Lac, Hipparcos results are in disagreement with the ground-based ones. $\mathrm{KK} \mathrm{Vel}$ is a singly-periodic star with $P=0.21570 \mathrm{~d}$ (Cousins 1982) or $P=$ $0.21569 \mathrm{~d}$ (Heynderickx 1992) for which Hipparcos gives $P=0.215644 \mathrm{~d}$. $\kappa$ Sco is a multiperiodic star with $P_{1}=0.1998303 \mathrm{~d}$ and $P_{2}=0.205430 \mathrm{~d}$ (Lomb \& Shobbrook 1975) while the period derived by Hipparcos is $P=0.2016988 \mathrm{~d}$. EN Lac is a multiperiodic star for which the Hipparcos period $P=0.171203 \mathrm{~d}$ is spurious; see Jerzykiewicz \& Pigulski (1999). Because Hipparcos observations form a homogeneous body of data, it is not clear why in these three cases Hipparcos results do not confirm periods from ground-based observations. The work on this problem is in progress.

\section{Periods of SPB Stars}

Hipparcos discovered 72 SPB stars (Waelkens et al. 1998). We examined the Hipparcos data in order to find out if they exhibit multiperiodicity. The results are displayed in Fig. 1 where HIP 18216 shows more than one frequency, while for HIP 34000 the power spectrum of prewhitened data exhibits nothing but noise. In the amplitude spectrum (not shown), the noise is below $0.003 \mathrm{mag}$. Because the only frequency of HIP 34000 is $0.60541 \mathrm{~d}^{-1}$, we suspect that its light variation may be due to ellipsoidal variability instead of pulsations.

\footnotetext{
${ }^{1}$ Based on data from the Hipparcos astrometry satellite.
} 


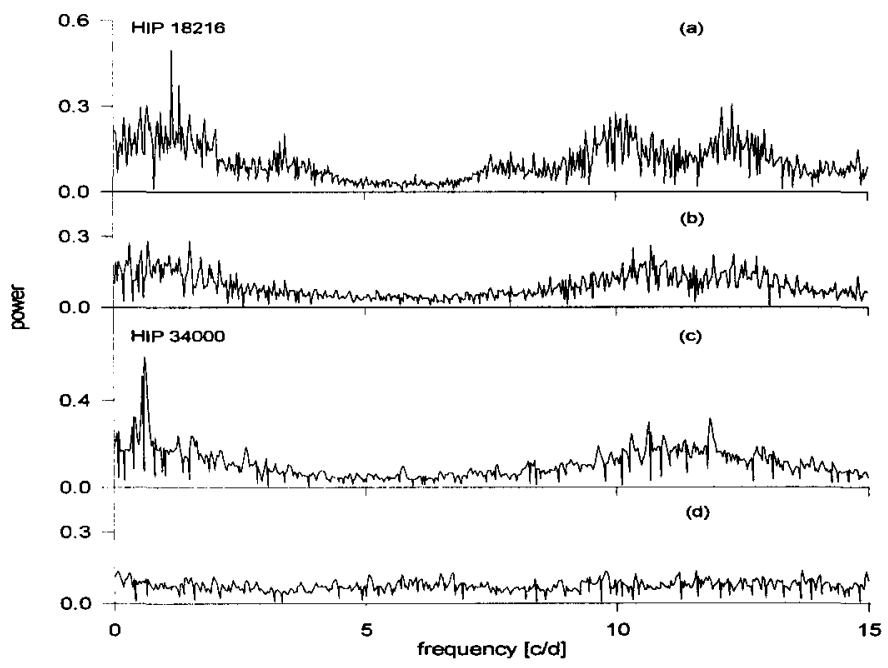

Figure 1. The two upper panels show: (a) power spectrum of HIP 18216 with the highest peak at $1.15691 \mathrm{~d}^{-1}$; (b) power spectrum of the data prewhitened with this frequency and with the highest peak at $1.52083 \mathrm{~d}^{-1}$. The two bottom panels show: (c) power spectrum of HIP 34000 with the highest peak at $0.60541 \mathrm{~d}^{-1} ;(d)$ power spectrum of data prewhitened with this frequency.

\section{Conclusions}

In most cases the periods of $\beta$ Cephei stars determined from the Hipparcos data agree with the ground-based ones. In three cases it is impossible to obtain even the main period of pulsation. As for SPB stars, we show that not all of them are multiperiodic variables. The possibility that the singly-periodic ones are in fact close binaries should be kept in mind.

Acknowledgments. An LOC grant is gratefully acknowledged. This work was supported by the Wrocław University grants 2041/W/IA/99 and $2313 /$ W/IA/99.

\section{References}

Cousins, A. W. J. 1982, IBVS, No. 2158

Heynderickx, D. 1992, A\&AS, 96, 207

Jerzykiewicz, M. \& Pigulski, A. 1999, MNRAS, in press

Lomb, N. R. \& Shobbrook, R. R. 1975, MNRAS, 173, 709

Sterken, C. \& Jerzykiewicz, M. 1993, Space Sci. Rev., 62, 95

Waelkens, C., Aerts, C., Kestens, E., Grenon, M., \& Eyer, L. 1998, A\&A, 330, 215 